eines „Inception Impact Assessment“ ist im ersten Quartal 2021 ein Vorschlag für einen künftigen KI-Rechtsrahmen $\mathrm{zu}$ erwarten, der nach heutigem Stand u.a. eine Revision der Produkthaftungsrichtlinie sowie Anforderungen an die Entwicklung und den Einsatz rechtmäßiger und vertrauenswürdiger KI umfassen wird ${ }^{93}$. Es bleibt zu hoffen, dass der europäische Gesetzgeber den Spagat zwischen Innovationsförderung und Ordnungspolitik für KI i.S. der Innovation nutzt und Anpassungen des geltenden Rechtsrahmens an die Erfordernisse des Einsatzes von KI in der Medizin auf die absolut notwendigen Eingriffe beschränkt, ohne in einer innovationshemmenden Überregulierung zu enden. Die Hoffnung wird u. a. dadurch gestärkt, dass die Kommission jüngst ankündigte, den Einsatz einer KIbasierten Software in zehn Krankenhäusern in Europa zu fördern, die in weniger als einer Minute CT Scans auf eine COVID-19 Erkrankung analysiert ${ }^{94}$.
Mit dem deutschen Ratsvorsitz im zweiten Halbjahr 2020 wird auch die Bundesregierung bei der Förderung von KI eine wichtige Rolle spielen. So steht u. a. die Entwicklung eines digitalen Frühwarnsystems auf dem Programm, um noch besser auf künftige der COVID-19 Pandemie vergleichbare Herausforderungen vorbereitet und handlungsfähig zu sein ${ }^{95}$.

93) S. https://ec.europa.eu/info/law/better-regulation/have-your-say/ initiatives/12527-Requirements-for-Artificial-Intelligence, Zugriff am 23.8.2020.

94) Vgl. Kommission, Pressebericht v. 19.5.2020, abrufbar unter https:// ec.europa.eu/digital-single-market/en/news/using-ai-fast-and-effectively-diagnose-covid-19-hospitals, Zugriff am 23.8.2020.

95) Programm des deutschen Vorsitzes im Rat der EU, S. 5, abrufbar unter https://www.eu2020.de/blob/2360248/e0312c50f91 0931819ab67f630d15b2f/pdf-programm-en-data.pdf, Zugriff am 23. 8.2020 .

\title{
Masernimpfpflicht, Maskenpflicht und unrichtige Gesundheitszeugnisse
}

\section{Sven Henseler}

\begin{abstract}
Masernimpfpflicht und das Tragen einer Mund-Nasen-Bedeckung können aus medizinischen Gründen suspendiert werden. Stellen $\ddot{A r z t}{ }^{\star}$ innen diesbezüglich ein unrichtiges Gesundheitszeugnis aus, machen sie sich nach $\$ 278$ StGB strafbar. Sie handeln sorgfaltsund standeswidrig, daher kann das Verhalten auch Anknüpfungspunkt für eine fahrlässige Körperverletzung (bzw. Tötung) sein und zum Widerruf der Approbation führen.
\end{abstract}

\section{Einleitung}

Mit dem Gesetz für den Schutz vor Masern und zur Stärkung der Impfprävention (Masernschutzgesetz) wurden mit Wirkung zum 1.3.2020 ${ }^{1}$ Personen verpflichtet, die nach dem 31.12.1970 geboren sind und in einer Gemeinschaftseinrichtung $^{2}$ betreut ${ }^{3}$ werden oder tätig sind ${ }^{4}$, einen ausreichenden Impfschutz gegen Masern oder $\mathrm{ab}$ der Vollendung des ersten Lebensjahres eine Immunität gegen Masern aufzuweisen $(\$ 20$ Abs. 8 S. 1 IfSG $)^{5}$. Eine Ausnahme gilt für Personen, die aufgrund einer medizinischen Kontraindikation nicht geimpft werden können (\$20 Abs. 8 S. 4 IfSG).

Die Reaktionen im Internet belegen, dass das Gesetz Wasser auf die Mühlen von Impfgegnern, Impfkritikern, Verteidigern der Menschenwürde und selbsternannten Medizinjournalisten war $^{6}$. In Foren wird sich über Umgehungsstrategien ausgetauscht. In einem Eilverfahren hat das $\mathrm{BVerfG}^{7}$ einen Antrag auf Erlass einer einstweiligen Anordnung ${ }^{8}$ abgelehnt. Dies sagt noch nichts über die Vereinbarkeit mit dem Verfassungsrecht aus ${ }^{9}$, sondern stellt lediglich fest, dass das Interesse an einer vorläufigen Aussetzung des Gesetzes hinter den Interessen an einer vorläufigen Weitergeltung zurücktritt ${ }^{10}$.

Rechtsanwalt Prof. Dr. iur. Sven Henseler,

Diplom-Finanzwirt (FH),

Inhaber einer Qualifikationsprofessur für Strafrecht,

Strafprozessrecht, Wirtschaftsstrafrecht und Steuerstrafrecht,

EBS Universität für Wirtschaft und Recht,

Gustav-Stresemann-Ring 3, 65189 Wiesbaden, Deutschland
Die Corona-Pandemie hat auch zu Vorschriften zum Schutz von anderen geführt. In den Landesverordnungen finden sich Regelungen über das verpflichtende Tragen von Mund-Nasen-Bedeckungen in bestimmten Situationen (Fahrzeuge des öffentlichen Personennah- und -fernverkehrs, Verkaufsstätten und ähnliche Einrichtungen, etc. ${ }^{11}$. Ausgenommen sind Personen, die aufgrund einer gesundheitlichen Beeinträchtigung oder Behinderung kei-

1) BGBl. I S. 148 .

2) $\$ 33$ Nr. 1 bis 3 IfSG: Kindertageseinrichtungen und Kinderhorte, nach $\$ 43$ Abs. 1 SGB VIII erlaubnispflichtige Kindertagespflege, Schulen und sonstige Ausbildungseinrichtungen.

3) Die Verpflichtung gilt auch für Personen, die bereits vier Wochen in einem Heim ( $\$ 33 \mathrm{Nr} .4$ IfSG) betreut werden oder in einer Einrichtungen zur gemeinschaftlichen Unterbringung von Asylbewerbern, vollziehbar Ausreisepflichtigen, Flüchtlingen und Spätaussiedlern (\$36 Abs. $1 \mathrm{Nr}$. 4 IfSG) untergebracht sind.

4) Auch Tätige in weiteren Einrichtungen nach den $\$ 23$ Abs. 1 und $\$ 36$ Abs. 1 IfSG.

5) Ausführlich zu den Einrichtungen und Pflichten: Rixen, NJW 2020, 647, 647f.

6) Quelle: Internet oder Youtube, um die Zitierung der selbsternannten „Experten“ aufzugreifen.

7) BVerfG, NJW 2020, $1946 \mathrm{ff}$.

8) Antrag und Verfassungsbeschwerde betreffen ein Elternpaar und ihre beiden ungeimpften Kinder, bei denen weder eine Kontraindikation noch eine Immunität vorliegen.

9) BVerfG, NJW 2020, 1946, 1947: „Die Verfassungsbeschwerde ist zumindest nicht von vornherein unzulässig oder offensichtlich unbegründet".

10) Zur Frage der Verfassungsmäßigkeit des $\$ 20$ Abs. 8 S. 3 IfSG: Schaks, MedR 2020, $201 \mathrm{ff}$; vgl. zur Verfassungsmäßigkeit insgesamt die Verweise in Fn. 53 bei Rixen, NJW 2020, 647, 651.

11) Beispielsweise: $₫ 1$ Abs. 6 S. 1 und $₫ 3$ Abs. 1 S. 1 Verordnung zur Beschränkung von sozialen Kontakten und des Betriebes von Einrichtungen und von Angeboten aufgrund der Corona-Pandemie (Corona-Kontakt-und Betriebsbeschränkungsverordnung) i.d.F der am 6.7.2020 in Kraft tretenden Änderungen durch Art. 3 der Fünfzehnten Verordnung zur Anpassung der Verordnungen zur Bekämpfung des Corona-Virus v. 1.7.2020 (GVB1. S. 473). 
ne Mund-Nasen-Bedeckung tragen können ${ }^{12}$. Es mehren sich Nachrichten über Verstöße gegen die Verpflichtung.

\section{Strafbarkeit nach $₫ 278$ StGB}

Beide Regelungskomplexe sehen demnach eine Ausnahme für Erkrankte bzw. Gefährdete vor. Ärzt^innen kommt daher eine wichtige Funktion im Hinblick auf eine Ausnahmebescheinigung zu. In der Vergangenheit ${ }^{13}$ wurde bereits mehrfach von Fällen sog. Gefälligkeitsatteste berichtet ${ }^{14}$ und ein bislang nicht geschätztes Dunkelfeld angesprochen. Im stressigen Ausbildungs- und Arbeitsleben seien Gefälligkeitsatteste wohl keine Seltenheit ${ }^{15}$. Die Gründe sind vielfältig: Es wird eine Arbeitsunfähigkeitsbescheinigung zur Verlängerung des Wochenendes gebraucht, ein Rücktritt von einer Prüfung bedarf eines tragfähigen Grundes, die Stornierungskosten für die Urlaubsreise sollen umgangen werden oder die Verhandlungsunfähigkeit eines Angeklagten muss belegt werden.

Die Ausstellung eines ärztlichen Zeugnisses zwecks „Freistellung von der Impflicht und anderen Maßnahmen der spezifischen Prophylaxe"16 oder zur Befreiung von der Verpflichtung zum Tragen einer Mund-Nasen-Bedeckung hat jedoch eine andere „Qualität", da hierdurch der Schutz, den diese Maßnahmen bewirken sollen, ausgehöhlt wird. Geht man der Frage der Strafbarkeit eines solchen Verhaltens nach, gelangt man zur Vorschrift des $\$ 278$ StGB „Ausstellen unrichtiger Gesundheitszeugnisse". Danach werden Ärzte und andere approbierte Medizinalpersonen ${ }^{17}$, welche ein unrichtiges Zeugnis über den Gesundheitszustand eines Menschen zum Gebrauch bei einer Behörde oder Versicherungsgesellschaft wider besseres Wissen ausstellen, mit Freiheitsstrafe bis zu zwei Jahren oder mit Geldstrafe bestraft. Bei dem Urkundsdelikt des $\$ 278$ StGB geht es damit um formell echte, aber inhaltlich unrichtige Gesundheitszeugnisse (eine bei der Urkundenfälschung i. S. v. $\$ 267 \mathrm{StGB}$ straflose sog. schriftliche Lüge) ${ }^{18}$. Unrichtig ist ein Gesundheitszeugnis, wenn eine in ihm enthaltene Aussage (über Befundtatsachen oder sachverständige Schlussfolgerungen) mindestens in einem wesentlichen Punkt nicht mit der Wirklichkeit übereinstimmt ${ }^{19}$. $\mathrm{Ob}$ ein Zeugnis auch dann unrichtig ist, wenn das Ergebnis ohne ärztliche Untersuchung bescheinigt wird, wird in der Literatur unterschiedlich gesehen ${ }^{20}$. Allein die unwahre Erklärung, dass bestimmte medizinische Untersuchungen an einem Menschen vorgenommen worden sind, reiche für $\$ 278 \mathrm{StGB}$ nicht aus, da sich dies nicht auf den Gesundheitszustand beziehe ${ }^{21}$. Nach anderer Ansicht sei ein ärztliches Gesundheitszeugnis unrichtig, durch das wahrheitswidrig eine Untersuchung behauptet oder auf eine solche Bezug genommen wird, die tatsächlich nicht stattgefunden hat ${ }^{22}$. Für die vorliegende Konstellation (Bescheinigung einer medizinisch notwendigen Ausnahme von der Masernimpflicht bzw. der Mund-Nase-Bedeckungspflicht) spielt der Streit ${ }^{23}$ keine Rolle. Ein Attest wird hierbei ggf. zwar ohne Vornahme einer Untersuchung ausgestellt, der mitgeteilte Befund entspricht aber auch nicht der Wirklichkeit. Nicht nur eine Mitteilung über Untersuchungen ist unrichtig, sondern auch der Gesundheitszustand wird unrichtig widergegeben ${ }^{24}$. Festgehalten werden kann, dass jedes Attest über eine medizinische Notwendigkeit auf die Masernschutzimpfung bzw. das Tragen einer Mund-Nasen-Bedeckung zu verzichten, was nicht der Wahrheit entspricht, ein unrichtiges $\mathrm{Ge}$ sundheitszeugnis darstellt.

Adressaten des beabsichtigten Gebrauchs sind inländische oder ausländische Behörden (auch Gerichte ${ }^{25}$ ) sowie private oder öffentlich-rechtliche Versicherungsgesellschaften. „Unter einer Behörde versteht man im allgemeinen eine in den Organismus der Staatsverwaltung eingeordnete, organisatorische Einheit von Personen und sächlichen Mitteln, die mit einer gewissen Selbständigkeit ausgestattet dazu berufen ist, unter öffentlicher Autorität für die Erreichung der Zwecke des Staates oder von ihm geförderter Zwecke tätig zu sein" ${ }^{26}$. Gemäß $\$ 20$ Abs. 9 S. 1 IfSG ist der Nachweis der Leitung der jeweiligen Einrichtung vorzulegen. Nach Satz 4 hat die Einrichtung das Gesundheitsamt, in dessen Bezirk sich die Einrichtung befindet, unverzüglich darüber zu benachrichtigen und dem Gesundheitsamt personenbezogene Angaben zu übermitteln. Die Gesundheitsämter ${ }^{27}$ sind Behörden nach $₫ 278$ i. V.m. $₫ 11 \mathrm{Nr} .7$ StGB. Mithin dient eine Bescheinigung über eine bestehende Kontraindikation ${ }^{28}(\mathbb{} 20$ Abs. 9 S. 1 Nr. 2 IfSG) zum Gebrauch bei einer Behörde.

Tathandlung ist das Ausstellen des Zeugnisses. Nach verbreiteter Ansicht soll dazu auch die Begebung in den Rechtsverkehr, etwa durch Übergabe an die Assistenz oder einen Empfänger gehören ${ }^{29}$. Nach anderer Ansicht soll die Ausstellung ausreichen, da der Gesetzestext keinen anderen Anknüpfungspunkt enthalte ${ }^{30}$. Führt man sich den Zweck der Norm vor Augen, nämlich die Wahrheit ärztlicher Gesundheitszeugnisse zu gewährleisten, um dadurch

12) Vgl. z.B. $\$ 1$ Abs. 4 S. 3 der obigen (Fn. 3) Verordnung.

13) So schon 1911: Hafter, ZStW 32, 271. Eisenmenger/Betz, DÄB1. 1993, A 126.

14) May, DÄB1. 2010, A 137 zu Attesten von Studierenden im Zusammenhang mit Prüfungen; Rühl, Bay. ÄBl. 2011, 650 f. zu Attesten von Schülern; Fenger/Gesenhues, DÄB1. 2009, A $1506 \mathrm{f}$.

15) Schroth, in: Roxin/Schroth, Handbuch des Medizinstrafrechts, 4. Aufl. 2010, Vorbemerkung II.5.

16) So die Bezeichnung eines Attestes in einem Fernsehbericht, wohl bezugnehmend auf die Überschrift des $\$ 20$ IfSG.

17) Ärzte und Zahnärzte $(\$ \subseteq 2,10,14 \mathrm{BÄO})$ und Angehörige sonstiger Heilberufe, deren Ausbildung staatlich geregelt ist und mit einer Staatsprüfung abgeschlossen wird (Erb, in: MüKo/StGB, 3. Aufl. 2019, \277, Rdnr. 3). Heilpraktiker fallen nicht hierunter (Puppe/Schumann, in: Kindhäuser/Neumann/Paeffen, Strafgesetzbuch, 5. Aufl. 2017, \$277, Rdnr. 5 m.w. N.). Die gegenteilige Ansicht (Heine/Schuster, in: Schönke/Schröder, Strafgesetzbuch, 30. Aufl. 2019, \$277, Rdnr. 3) verkennt die Rolle des Heilpraktikers im medizinischen Bereich. Die Genehmigung ( $\$ 2$ HeilprG) wird nur dann nicht erteilt, wenn der Antragsteller eine Gefahr für die Gesundheit der Bevölkerung oder für die ihn aufsuchenden Patientinnen und Patienten bedeuten würde ( $\$ 2$ Abs. 1i) HeilprGDV 1).

18) Ulsenheimer, in: Laufs/Kern/Rehborn, Handbuch des Arztrechts, 5. Aufl. 2019, §156, Rdnr. 1.

19) Erb, in: MüKo/StGB, 3. Aufl. 2019, \$278, Rdnr. 4.

20) Zuck/Gokel, in: Quaas/Zuck/Clemens, Medizinrecht, 4. Aufl. 2018, \$72, Rdnr. 20.

21) Hoyer, in: Wolter, Systematischer Kommentar zum Strafgesetzbuch, 9. Aufl. 2019, \$278, Rdnr. 2.

22) Gercke, MedR 2008, 592, 593

23) Vgl. zum Streit: Wolfslast, in: Schünemann/Achenbach/Bottke/Haffke/Rudolphi, FS f. Roxin, 2011, S. 1121, $1122 \mathrm{ff}$.

24) Ob ein Gesundheitszeugnis auch ohne körperliche Untersuchung ausgestellt werden kann, ist Sache des Einzelfalles. Es können Situationen gegeben sein, bei denen es medizinisch vertretbar oder gar geboten ist, eine unmittelbare Untersuchung zu unterlassen (vgl. Gercke, MedR 2008, 592, 593).

25) $\$ 11$ Abs. 1 Nr. 7 StGB.

26) BVerfGE 10, 20, 48; BGH, NVwZ 2010, 919, 920.

27) RGSt 74, 229, 231; Ulsenheimer, in: Laufs/Kern/Rehborn, Handbuch des Arztrechts, 5. Aufl. 2019, $\$ 278$, Rdnr. 4 mit Verweis auf das Urteil des RG.

28) Als medizinische Kontraindikationen zur MMR-Impfung gelten im Allgemeinen: akutes Fieber $\left(>38,5^{\circ} \mathrm{C}\right)$ oder eine akute schwere Erkrankung; Schwangerschaft; bestimmte schwere Einschränkungen des Immunsystems; bekannte Allergien gegen Bestandteile des Impfstoffs (https://www.rki.de/SharedDocs/FAQ/Impfen/MMR/Masernimpfung/FAQ-Liste_ Masernimpfung.html\#FAQId13739262, zuletzt aufgerufen am 23. 10.2020)

29) Wittig, in: Satzger/Schluckebier/Widmaier, Strafgesetzbuch, 4. Aufl. 2018, \$278, Rdnr. 7 m. w. N.

30) Erb, in: MüKo/StGB, 3. Aufl. 2019, \$278, Rdnr. 5. 
die Dispositionsfreiheit der darauf besonders angewiesenen Täuschungsadressaten ${ }^{31}$ bzw. darüber hinaus sogar die Sicherheit und Zuverlässigkeit des Rechtsverkehrs mit Gesundheitszeugnissen $^{32} \mathrm{zu}$ schützen, reicht die Ausstellung aus. Jedes unrichtige Gesundheitszeugnis beeinträchtigt das Vertrauen in die Wahrheit ärztlicher Atteste bzw. Gutachten. Als Korrektiv für die frühe Tatvollendung dient die Einschränkung, dass das Zeugnis zum Gebrauch bei einer Behörde oder Versicherungsgesellschaft ausgestellt wird; eine weitergehende Einschränkung ist im Gesetz nicht formuliert und auch nicht notwendig. Praktische Bedeutung hat dieses Problem für die vorliegenden Konstellationen nicht, da die unrichtigen Zeugnisse in den Rechtsverkehr gelangt sind.

Der subjektive Tatbestand setzt die positive Kenntnis der Unwahrheit der attestierten Tatsachen (Handeln wider besseres Wissen) voraus ${ }^{33}$. Bezogen auf die Bestimmung (Gebrauch) des Gesundheitszeugnisses reicht es aus, wenn der Täter die Möglichkeit des Gebrauchs - im Zusammenhang mit der Beurteilung des Gesundheitszustands einer Person - sieht und diesen billigend in Kauf nimmt ${ }^{34}$. Die Fälle unrichtiger Bescheinigung einer medizinisch notwendigen Ausnahme als sog. Gefälligkeitsattests erfolgen absichtlich, sodass der subjektive Tatbestand gegeben ist. Rechtfertigungsgründe für das Ausstellen eines unrichtigen Gesundheitszeugnisses sind nicht ersichtlich. Wer als Ärztin oder Arzt eine unrichtige Bescheinigung über eine bestehende Kontraindikation in Bezug auf die Masernimpfung ${ }^{35}$ oder das Tragen einer Mund-Nasen-Bedeckung ausstellt, macht sich wegen $\$ 278$ StGB strafbar.

\section{Weitere Strafbarkeiten}

Wurde jemand in unzulässiger Weise von der Masernimpfpflicht ausgenommen und steckt dann in einer Einrichtung eine andere Person mit Masern an - oder wurde jemand zu Unrecht von der Pflicht zum Tragen einer Mund-Nasendeckung befreit und steckt eine andere Person deswegen mit einer Krankheit an, kommt eine Strafbarkeit desjenigen, der das unrichtige Gesundheitszeugnis ausgestellt hat, wegen Körperverletzung in Betracht. Eine Krankheit stellt eine Schädigung der Gesundheit im Sinne der Körperverletzung (\$223 StGB) dar. In den meisten Fällen dürfte diese Ansteckung von den Ärzt $\star_{i n n e n}$ nicht gewollt gewesen sein, sodass keine vorsätzliche, sondern eine fahrlässige Körperverletzung ( $\$ 229$ StGB) vorliegen könnte. Das Ausstellen einer unrichtigen Gesundheitsbescheinigung trotz Kenntnis vom Fehlen einer medizinischen Kontraindikation entspricht nicht dem Verhalten, das von einem gewissenhaften und aufmerksamen Arzt aus berufsfachlicher Sicht seines Fachbereichs vorausgesetzt und erwartet wird $^{36}$ und stellt daher eine Sorgfaltspflichtverletzung dar. Gerade diese Sorgfaltspflichtverletzung hat es ermöglicht, dass jemand ohne notwendigen Schutz (Impfung oder Mund-Nasen-Bedeckung) eine andere Person angesteckt hat. Hierin erfüllt sich auch gerade das Risiko, das der Täter durch seine Handlung bewirkt hat. Eine fährlässige Körperverletzung liegt daher vor. Verstirbt jemand an den Folgen der Erkrankung liegt eine fahrlässige Tötung (\$222 StGB) vor.

\section{Berufsrechtliche Folgen}

Berufsrechtliche Folge kann der Verlust der Approbation sein. Die Approbation ist zu widerrufen, wenn sich nachträglich Unwürdigkeit oder Unzuverlässigkeit zur Ausübung des ärztlichen Berufs ergeben hat ( $\$ \int 5$ Abs. 2 S. 1 i.V.m. $\$ 3$ Abs. 1 S. 1 Nr. 2 BÄO). Unwürdig ist, wer durch sein Verhalten das zur Ausübung des ärztlichen Berufes erforderliche Ansehen und Vertrauen bei der Bevölkerung nicht besitzt; unzuverlässig ist, wer nach seiner
Gesamtpersönlichkeit keine ausreichende Gewähr für eine ordnungsgemäße Berufsausübung bietet ${ }^{37}$. Für die Unwürdigkeit wird ein schwerwiegendes Fehlverhalten des Arztes verlangt, das bei Würdigung aller Umstände seine weitere Berufsausübung im maßgeblichen Zeitpunkt untragbar erscheinen lässt ${ }^{38}$. Wer sich dem wissenschaftlichen Diskurs verschließt - seinen Standpunkt hinsichtlich der Notwendigkeit von Schutzmaßnahmen (Impfung, Maskenpflicht, etc.) nicht offenlegt, der bietet in der Regel nicht die Gewähr für die Ausübung des Arztberufes ${ }^{39}$. Ungeachtet der verfassungsrechtlichen Bedenken gegen die Vorschrift über die Masernimpfpflicht, liegt es nicht in der Hand von Ärzt`innen einen - aus ihrer Sicht - verfassungsmäßigen Zustand herzustellen.

Sofern Ärztekammern sich an einem Verfahren gegen approbierte Mediziner*innen gehindert sehen, da die Informationen ohne Entbindung von der ärztlichen Schweigepflicht nicht erlangt werden können, sollten die Eltern ausdrücklich aufgefordert werden, den Arzt von der Schweigepflicht zu entbinden. Kommen die Eltern dieser Aufforderung nicht nach, sollte der Sachverhalt, der den Verdacht nach $\$ 278$ StGB begründet, bei der zuständigen Staatsanwaltschaft angezeigt werden. Im Rahmen des Ermittlungsverfahrens wird die Staatsanwaltschaft prüfen, ob eine Durchsuchung ( $\$ 102$ StPO) beantragt werden kann ${ }^{40}$ und sollte oder ob eine Entbindung von der Schweigepflicht auf andere Art erfolgen kann. Ausgangspunkt ist die Überlegung, dass nicht nur eine Strafbarkeit der Ärzt*innen gemäß $\int 278$ StGB in Betracht kommt, sondern dass die Eltern den Arzt zur unrichtigen Ausstellung überredet haben. Wird der Tatentschluss zu $\$ 278$ StGB durch die Eltern hervorgerufen, liegt hierin eine Anstiftung ( $\$ 26 \mathrm{StGB})$. Die Eltern können sich daher selbst strafbar gemacht haben ${ }^{41}$. Eine falsche ärztliche Bescheinigung (Vorliegen einer Kontraindikation) gefährdet das Kind, weshalb ein Interessenkonflikt zwischen elterlicher Sorge und Wohl des Kindes vorliegt. Für die Entbindung von der Schweigepflicht kann daher ein Ergänzungspfleger (S\$1629 Abs. 2 S. 3, 1796 Abs. 1, 1909 BGB) bestellt werden.

31) Puppe/Schumann, in: Kindhäuser/Neumann/Paeffen, Strafgesetzbuch, 5. Aufl. 2017, $\$ 278$, Rdnr. 2 m. w. N

32) Wittig, in: Satzger/Schluckebier/Widmaier, Strafgesetzbuch, 4. Aufl. 2018, \$278, Rdnr. 2 m.w. N.

33) Puppe/Schumann, in: Kindhäuser/Neumann/Paeffgen, Strafgesetzbuch, 5. Aufl. 2017, \$278, Rdnr. 3.

34) Erb, in: MüKo/StGB, 3. Aufl. 2019, \$278, Rdnr. 6.

35) Im Allgemeinen keine Kontraindikation zur MMR-Impfung sind zum Beispiel: banale Infekte, auch wenn sie mit subfebrilen Temperaturen $\left(<38,5^{\circ} \mathrm{C}\right)$ einhergehen und eine ,erhöhte Infektanfälligkeit“", Behandlung mit Antibiotika; Ekzem u.a. Dermatosen; lokalisierte Hautinfektionen; chronische Erkrankungen wie Asthma; Behandlung mit niedrigen Dosen von Kortikosteroiden oder lokal angewendeten steroidhaltigen Präparaten, Krampfanfälle in der Vorgeschichte oder bei Familienmitgliedern; eine Hühnereiweißallergie (in den allermeisten Fällen); ein möglicher Kontakt der zu impfenden Person zu Personen mit ansteckenden Krankheiten; Schwangerschaft der Mutter des zu impfenden Kindes und stillende Frauen - sie können alle notwendigen Impfungen erhalten außer einer Impfung gegen Gelbfieber (https://www.rki.de/SharedDocs/ FAQ/Impfen/MMR/Masernimpfung/FAQ-Liste_Masernimpfung.html\#FAQId13739262, zuletzt aufgerufen am 23.10.2020).

36) $\mathrm{BGH}, \mathrm{NJW} 1995,776,777$.

37) Heinz/Haage, in: Bundesärzteordnung, $\$ 3$, Rdnr. 4 m.w. N.

38) BVerwG, Beschl. v. 28.1.2003 - 3 B 149/02 -, BeckRS 2003, 21187.

39) Ähnlich, wer die Schulmedizin als solche ablehnt (vgl. VG Frankfurt, Urt. v. 7.2.2017 - 4 K 3468/16.F).

40) Vgl. zu rechtlichen Problemen bei der Durchsuchung von Arztpraxen: Röß $\beta$, NZWiSt 2018, $483 \mathrm{ff}$.

41) $\ 52$ Abs. 2 S. 2 StPO sieht für den Fall des Zeugnisverweigerungsrechts (des Kindes) vor, dass der gesetzliche Vertreter, der selbst Beschuldigter ist, über die Ausübung des Zeugnisverweigerungsrechts nicht entscheiden kann. 
Wurden die unrichtigen Gesundheitszeugnisse ausgestellt und nicht abgerechnet, kommt ein Verstoß gegen die Liquidationspflicht in Betracht. Gemäß $\$ 12$ Abs. 1 S. 3 MBO-̈̈ dürfen Ärztinnen und Ärzte die Sätze nach der GOÄ nicht in unlauterer Weise unterschreiten. Auf die Unrichtigkeit des Gesundheitszeugnisses kann es nicht ankommen.

\section{Fazit}

Die Skepsis gegen die gesetzlichen Regelungen mag berechtigt sein. Gerade die Masernimpfpflicht sieht sich nicht nur dem Vorwurf der Verfassungswidrigkeit ausgesetzt, sondern kann auch die Frage nicht beantworten, wie das Ziel, den Impfschutz möglichst aller herzustellen, erreicht werden soll, wenn Ungeimpfte die Einrichtungen meiden. Dennoch haben diese Regeln Bestand. Wer sich eigenmächtig darüber hinwegsetzt und unrichtige Gesundheitszeugnisse ausstellt, macht sich nach $\$ 278$ StGB strafbar und im Fall einer (Masern-) Erkrankung mit schwerwiegenden - ggf. sogar tödlichen - Folgen grundsätzlich auch wegen (zumindest) fahrlässiger Körperverletzung - ggf. fahrlässiger Tötung - strafbar.

\section{Das Gesundheitswesen im Fokus der Investitionskontrolle}

\section{Laura Louca und Alexander Kopf}

\begin{abstract}
:
Der Beitrag befasst sich mit den unter dem Eindruck der Covid-19 Epidemie verschärften Regelungen zur Investitionskontrolle im Bereich des Gesundheitswesens. Gegenstand sind dabei zum einen die Einzelheiten der neu eingeführten Regelbeispiele der Außenwirtschaftsverordnung (AWV) im Kontext der Besonderheiten des Gesundheitsrechts. Darüber hinaus wird ein Ausblick auf die weitere Entwicklung sowie die Bedeutung für betroffene Unternehmen gegeben.
\end{abstract}

\section{Verschärfung der Investitionskontrolle im Zuge der Covid-19 Epidemie}

Fragen des Außenwirtschaftsrechts und der Investitionskontrolle haben bisher im Medizin- und Gesundheitsrecht eine eher untergeordnete Rolle gespielt. Mit der Covid-19 Epidemie und den damit zu Tage getretenen Versorgungsproblemen hat sich dies schlagartig geändert. Als Ergebnis ist im Zuge der jüngsten Reform des Außenwirtschaftsrechts das Investitionsprüfrecht des Bundeswirtschaftsministeriums (BMWi) erheblich gestärkt worden. Mit der am 3.6.2020 in Kraft getretenen 15. Verordnung zur Änderung der Außenwirtschaftsverordnung (15. AWV-Novelle) und der am 17.7.2020 in Kraft getretenen Änderung des Außenwirtschaftsgesetzes wurde der Prüfungsmaßstab vertieft und der Anwendungsbereich der Investitionskontrolle insbesondere im Gesundheitssektor erweitert. Am 29.10. 2020 ist die 16. Verordnung zur Änderung der AuBenwirtschaftsverordnung (16. AWV-Novelle) in Kraft getreten. Damit werden die im Juli 2020 im Einklang mit der EU-Screening-Verordnung beschlossenen Gesetzesänderungen in die Verordnung eingefügt.

Das Ziel der Investitionskontrolle besteht darin, die Übernahme und Steuerung inländischer Unternehmen bestimmter sensibler Wirtschaftsbereiche durch ausländische Investoren zu regulieren. Die Rechtsgrundlage hierfür findet sich

Rechtsanwältin Dr. iur. Laura Louca,

BLOMSTEIN Partnerschaft von Rechtsanwälten mbB,

Oranienburger Straße 66, 10117 Berlin, Deutschland

Rechtsanwalt Dr. iur. Alexander Kopf,

D+B Rechtsanwälte Partnerschaft mbB,

Kurfürstendamm 195, 10707 Berlin, Deutschland im Außenwirtschaftsgesetz (AWG) und wird in ihren Einzelheiten in der Außenwirtschaftsverordnung (AWV) näher ausgestaltet. Daneben bestehen in Form der EU-ScreeningVerordnung auch europarechtliche Vorgaben. Der Gesetzgeber knüpft die Kontrollbefugnis des BMWi generalklauselartig an eine „Beeinträchtigung der öffentlichen Ordnung und Sicherheit" (\$5 AWG n.F., $\ 55$ Abs. 1 S. 1 AWV n. F.).

Die Investitionsprüfung ist so strukturiert, dass grundsätzlich bei jedem Erwerb eines inländischen Unternehmens die potenzielle Beeinträchtigung der öffentlichen Sicherheit und Ordnung geprüft werden kann. Im sektorübergreifenden Bereich prüft das BMWi, ob die weiteren Voraussetzungen der $\$ \int 55 \mathrm{ff}$. AWV vorliegen, insbesondere ob ein Unternehmen aus dem Regelkatalog des $₫ 55$ Abs. 1 S. 2 AWV n. F. erworben wird und die Beteiligungsschwellen des $₫ 56$ AWV erfüllt sind.

Sofern der Erwerb eines inländischen Unternehmens den Tatbestand des $₫ 55$ Abs. 1 AWV erfüllt, muss die Transaktion gegenüber dem BMWi angezeigt werden ( $\$ 55$ Abs. 4 S. 1 AWV n.F.). Aus dem Prüfungsrecht des BMWi folgt also eine Anzeigepflicht. Das bedeutet, dass die Beteiligten eines Unternehmenskaufs oder einer Beteiligung diese Frage selbst prüfen und aufgrund dieser Prüfung vor der Transaktion eine Entscheidung über eine Meldung an das BMWi treffen müssen. $\int 55 \mathrm{Abs}$. 1a AWV n. F. stellt klar, dass auch Asset Deals vom Erwerbsbegriff des $\$ 55$ Abs. 1 AWV n. F. erfasst werden.

\section{Erweiterung der AWG-Generalklausel}

Während nach $\$ 5$ AWG a.F. der (teilweise) Erwerb eines Unternehmens eine tatsächliche Gefährdung der öffentlichen Ordnung und Sicherheit darstellen musste, orientiert sich $\ 5$ Abs. 2 AWG n. F. am Vorbild der EU-ScreeningVerordnung. Die Neuregelung ermöglicht dem BMWi nunmehr einen Erwerb auch dann zu untersagen, wenn eine „,voraussichtliche Beeinträchtigung“ der öffentlichen Ordnung oder Sicherheit vorliegt. Eine tatsächliche Gefährdung ist demnach nicht mehr erforderlich. Vielmehr kann das BMWi vorausschauend im Sinne einer Prognoseentscheidung mögliche Beeinträchtigungen verhindern, indem es erwerbsbeschränkende Maßnahmen anordnet. Das kann sowohl in Form einer Untersagung des Erwerbs, als auch durch die Genehmigung unter Auflagen erfolgen. Der Gesetzgeber hat deutlich gemacht, dass der Fokus der Investitionsprüfung über (Landes-)Sicherheit, öffentliche Ordnung und kritische Infrastrukturen hinausgehen kann. 